\title{
Reactor physics modelling of accident tolerant fuel for LWRs using ANSWERS codes
}

\author{
Benjamin A. Lindley ${ }^{1 *}$, Dan Kotlyar ${ }^{2}$, Geoffrey T. Parks ${ }^{2}$, John N. Lillington ${ }^{1}$, and Bojan Petrovic ${ }^{3}$ \\ 1 Amec Foster Wheeler, Dorchester, UK \\ 2 Department of Engineering, University of Cambridge, Cambridge, UK \\ ${ }^{3}$ Georgia Institute of Technology, Georgia, USA
}

Received: 10 September 2015 / Received in final form: 5 February 2016 / Accepted: 15 February 2016 Published online: 25 March 2016

\begin{abstract}
The majority of nuclear reactors operating in the world today and similarly the majority of near-term new build reactors will be LWRs. These currently accommodate traditional $\mathrm{Zr}$ clad $\mathrm{UO}_{2} / \mathrm{PuO}_{2}$ fuel designs which have an excellent performance record for normal operation. However, the events at Fukushima culminated in significant hydrogen production and hydrogen explosions, resulting from high temperature $\mathrm{Zr} /$ steam interaction following core uncovering for an extended period. These events have resulted in increased emphasis towards developing more accident tolerant fuels (ATFs)-clad systems, particularly for current and near-term build LWRs. R\&D programmes are underway in the US and elsewhere to develop ATFs and the UK is engaging in these international programmes. Candidate advanced fuel materials include uranium nitride (UN) and uranium silicide $\left(\mathrm{U}_{3} \mathrm{Si}_{2}\right)$. Candidate cladding materials include advanced stainless steel (FeCrAl) and silicon carbide. The UK has a long history in industrial fuel manufacture and fabrication for a wide range of reactor systems including LWRs. This is supported by a national infrastructure to perform experimental and theoretical $R \& D$ in fuel performance, fuel transient behaviour and reactor physics. In this paper, an analysis of the Integral Inherently Safe LWR design $\left(\mathrm{I}^{2} \mathrm{~S}-\mathrm{LWR}\right)$, a reactor concept developed by an international collaboration led by the Georgia Institute of Technology, within a US DOE Nuclear Energy University Program (NEUP) Integrated Research Project (IRP) is considered. The analysis is performed using the ANSWERS reactor physics code WIMS and the EDF Energy core simulator PANTHER by researchers at the University of Cambridge. The $\mathrm{I}^{2} \mathrm{~S}-\mathrm{LWR}$ is an advanced $2850 \mathrm{MWt}$ integral PWR with inherent safety features. In order to enhance the safety features, the baseline fuel and cladding materials that were chosen for the $\mathrm{I}^{2} \mathrm{~S}-\mathrm{LWR}$ design are $\mathrm{U}_{3} \mathrm{Si}_{2}$ and advanced stainless steel respectively. In addition, the $\mathrm{I}^{2} \mathrm{~S}-\mathrm{LWR}$ design adopts an integral configuration and a fully passive decay heat removal system to provide indefinite cooling capability for a class of accidents. This paper presents the equilibrium cycle core design and reactor physics behaviour of the $\mathrm{I}^{2} \mathrm{~S}-\mathrm{LWR}$ with $\mathrm{U}_{3} \mathrm{Si}_{2}$ and the advanced steel cladding. The results were obtained using the traditional two-stage approach, in which homogenized macroscopic cross-section sets were generated by WIMS and applied in a full 3D core solution with PANTHER. The results obtained with WIMS/PANTHER were compared against the Monte Carlo Serpent code developed by VTT and previously reported results for the $\mathrm{I}^{2} \mathrm{~S}-\mathrm{LWR}$. The results were found to be in a good agreement (e.g. $<200 \mathrm{pcm}$ in reactivity) among the compared codes, giving confidence that the WIMS/PANTHER reactor physics package can be reliably used in modelling advanced LWR systems.
\end{abstract}

\section{Introduction}

The majority of nuclear reactors operating in the world today and similarly the majority of near-term new build reactors will be LWRs. These currently accommodate traditional $\mathrm{Zr}$ clad $\mathrm{UO}_{2} / \mathrm{Pu}$ fuel designs which have an excellent performance record for normal operation. However,

\footnotetext{
* e-mail: ben.lindley@amecfw.com
}

the events at Fukushima culminated in significant hydrogen production and hydrogen explosions, resulting from high temperature $\mathrm{Zr}$ /steam interaction following core uncovering for an extended period. These events have resulted in increased emphasis towards developing more accident tolerant fuels (ATFs), particularly for current and nearterm build LWRs.

Candidate advanced fuel materials include uranium nitride $(\mathrm{UN})$ and uranium silicide $\left(\mathrm{U}_{3} \mathrm{Si}_{2}\right)$, both of which have higher thermal conductivity than $\mathrm{UO}_{2}$, leading to 
improved margins under accident conditions, and also have the benefit of higher heavy metal density leading to the possibility of increased core heavy metal loading [1,2]. Candidate cladding materials include advanced stainless steel ( $\mathrm{FeCrAl})$, silicon carbide ( $\mathrm{SiC})$, and the possibility of adding a coating to Zircaloy clad [3]. Stainless steel cladding exhibits a lower oxidation rate under accident conditions than Zircaloy [4] and is relatively easy to fabricate [5], but has the disadvantage of introducing a large reactivity penalty [4]. SiC cladding can withstand much higher temperatures than Zircaloy, but is expensive and difficult to fabricate [5]. R\&D programmes are underway in the US and elsewhere to develop ATFs, encompassing fabrication and testing of $\mathrm{UN}, \mathrm{U}_{3} \mathrm{Si}_{2}, \mathrm{SiC}$ and coated $\mathrm{Zr}$ rods [6].

This paper presents the core analysis performed with the ANSWERS reactor physics code suite WIMS/PANTHER $[7,8]$ for the Integral Inherently Safe Light Water Reactor $\left(\mathrm{I}^{2} \mathrm{~S}-\mathrm{LWR}\right)$. The $\mathrm{I}^{2} \mathrm{~S}-L W R$ concept [9] is a Gen III+ large scale (i.e. $1 \mathrm{GWe}$ ) reactor. The design stage is being carried out by a consortium of universities (Michigan, Virginia Tech, Tennessee, Florida Institute of Technology, Idaho, Morehouse College, Brigham Young University, Cambridge, Politecnico di Milano, Zagreb), Idaho National Laboratory, Westinghouse and Southern Nuclear Company. The project is led by the Georgia Institute of Technology.

This innovative PWR includes: an integral primary circuit, a fully passive decay heat removal system that provides indefinite cooling capability, and the use of new materials. The types of materials that were originally chosen for this design include $\mathrm{U}_{3} \mathrm{Si}_{2}$ fuel pellets within advanced steel cladding.

The equilibrium cycle core analysis was performed using the WIMS/PANTHER codes and the results were verified in a code-to-code comparison. In the first stage, the 2D results obtained with WIMS [2] were compared against the Monte Carlo code Serpent [10], and a good agreement was observed. In the second stage, the full 3D core results obtained with the WIMS/PANTHER codes were compared with results form the literature for the $\mathrm{I}^{2} \mathrm{~S}-\mathrm{LWR}$ [11]. This cross-comparison of results provides enhanced confidence in the reliability and accuracy of the results.

\section{UK context for accident tolerant fuel}

The UK has a long history in industrial fuel manufacture and fabrication for a wide range of reactor systems including LWRs. This is supported by a national infrastructure to perform experimental and theoretical $\mathrm{R} \& \mathrm{D}$ in fuel performance, fuel transient behaviour and reactor physics.

The UK is seeking to engage with international programmes on ATF research to "strengthen international collaboration opportunities and establish the UK as a centre of expertise for advanced fuel fabrication $R \& D$, and consequently commercial manufacture of such fuels" [12]. Such fuels could be utilized in nuclear new build plants, and also potentially in small modular reactors (SMRs), in which the UK has expressed a strategic interest [13]. The UK
Nuclear Industry Research and Advisory Board (NIRAB) recently recommended that the UK perform research on manufacturing advanced cladding materials in order to enable future manufacture of ATF on a commercial scale [14]. Opportunities for ATF use are identified to include Generation III reactors and SMRs.

\section{Modelling of accident tolerant fuel with ANSWERS software}

The ANSWERS lattice code WIMS and core simulator PANTHER are used to support the operation of existing PWRs, including in the UK and Belgium [15]. WIMSPANTHER has recently been validated for analysis of partMOX-fuelled PWRs. In academia, WIMS and PANTHER have also been applied to a range of PWR configurations including SMRs [16], seed-blanket-fuelled PWRs [17,18], PWRs loaded with transuranic fuels $[19,20]$. Modelling of ATFs is a natural extension of these capabilities and can largely be performed using existing calculation routes.

Challenges of modeling ATFs include:

- validation of software for different fuel types. This includes validation of the relevant nuclear data libraries. For stainless steel, an extensive amount of validation has been performed as steel is commonly used in fast and thermal reactors. For other isotopes/elements, a reasonable amount of experimental data is available, but further validation may be required for use in new applications; - modelling of non-standard isotopes. An example is the presence of ${ }^{15} \mathrm{~N}$ in UN fuel. The most abundant isotope of nitrogen, ${ }^{14} \mathrm{~N}$, has a large $(\mathrm{n}, \mathrm{p})$ cross-section which adversely impacts the neutron economy. It is therefore commonly proposed to increase the ${ }^{15} \mathrm{~N}$ content of the nitrogen in the UN fuel through enrichment [1]. While limited experimental data on ${ }^{15} \mathrm{~N}$ cross-sections is available, it is not usually considered in isolation and hence further experimental validation may be necessary for thermal reactor applications;

- some candidate ATFs may have the capability to be driven to higher burnups than existing Zircaloy clad $\mathrm{UO}_{2}$ fuels. Both stainless steel [4] and $\mathrm{SiC}$ [21] have superior performance when irradiated compared to Zircaloy. This leads to the need to validate the reactor physics code for higher enrichments and high burnups, and account for a wider range of actinides.

WIMS10, the most recent release of WIMS, contains nuclear data for high burnup applications, including crosssections and delayed neutron fraction data for a wider range of isotopes including ${ }^{246} \mathrm{Cm},{ }^{247} \mathrm{Cm}$ and ${ }^{248} \mathrm{Cm}$. Use of higher enrichment fuel, being driven to high burnups, leads to increased reactivity swings, which requires use of novel burnable poison arrangements and core loading strategies [22]. PANTHER contains inbuilt multi-objective optimization algorithms which facilitate PWR [23] and VVER [24] core design. These have recently been applied to the non-standard case where PWRs are highly loaded with $\mathrm{Pu}$ $[25,26]$ and have been shown to facilitate low power peaking core design under challenging circumstances. 


\begin{tabular}{|c|c|c|c|c|c|c|}
\hline $3 x$ & $1 X$ & $\begin{array}{c}4.65 \\
\% \\
100 \mathrm{~B}\end{array}$ & $2 X$ & $1 X$ & $\begin{array}{c}4.65 \\
\% \\
84 \mathrm{~B}\end{array}$ & $2 X$ \\
\hline $1 X$ & $2 X$ & $1 X$ & $1 \mathrm{X}$ & $\begin{array}{c}4.45 \\
\% \\
156 \mathrm{~B}\end{array}$ & $\begin{array}{c}4.65 \\
\% \\
84 \mathrm{~B}\end{array}$ & $2 X$ \\
\hline $\begin{array}{c}4.65 \\
\% \\
100 B\end{array}$ & $1 X$ & $1 X$ & $1 X$ & $\begin{array}{c}4.45 \\
\% \\
84 \mathrm{~B}\end{array}$ & $2 X$ & \\
\hline $2 X$ & $1 X$ & $1 X$ & $1 X$ & $\begin{array}{c}4.45 \\
\% \\
84 \mathrm{~B}\end{array}$ & $2 X$ & \\
\hline $1 X$ & $\begin{array}{c}4.45 \\
\% \\
156 \mathrm{~B}\end{array}$ & $\begin{array}{c}4.45 \\
\% \\
84 \mathrm{~B}\end{array}$ & $\begin{array}{c}4.45 \\
\% \\
84 \mathrm{~B}\end{array}$ & $2 X$ & & \\
\hline $\begin{array}{c}4.65 \\
\% \\
84 \mathrm{~B}\end{array}$ & $\begin{array}{c}4.65 \\
\% \\
84 B\end{array}$ & $2 X$ & $2 X$ & & & \\
\hline $2 X$ & $2 X$ & & & & & \\
\hline
\end{tabular}

Fig. 1. $I^{2}$ S-LWR equilibrium cycle core loading pattern (bottom right quadrant of the core).

\section{Use of WIMS/PANTHER to model $I^{2}$ S-LWR}

\section{1 $\mathrm{I}^{2} \mathrm{~S}-\mathrm{LWR}$ core description}

The $\mathrm{I}^{2} \mathrm{~S}$-LWR core contains 121 assemblies with 144-in active fuel height as shown in Figure 1 . The $\mathrm{I}^{2} \mathrm{~S}-\mathrm{LWR}$ is designed to achieve $40 \%$ higher power rating than a typical 2-loop Westinghouse core ( 2850 MWt vs. $\sim 2000 \mathrm{MWt})$. The major modification to achieve this objective was transitioning from a typical $16 \times 16$ assembly array to a $19 \times 19$ square pitch lattice. The increased number of fuel rods in the $19 \times 19$ lattice counterbalances the higher power density in the $\mathrm{I}^{2} \mathrm{~S}-\mathrm{LWR}$ thereby benefitting DNB performance and, also thanks to the high thermal conductivity of $\mathrm{U}_{3} \mathrm{Si}_{2}$, fuel temperature. The larger number of fuel rods in the $19 \times 19$ lattice leads to approximately same average linear power, $5.8 \mathrm{~kW} / \mathrm{ft}$, and only about $3 \%$ higher heat flux at the rod surface, $62 \mathrm{~kW} / \mathrm{ft}^{2}$, for the $\mathrm{I}^{2} \mathrm{~S}$-LWR relative to a $5 \%$ uprated 4-loop PWR with $17 \times 17$ lattice. It must be pointed out that the $\mathrm{H} / \mathrm{HM}$ atomic ratio for the $19 \times 19$ is lower, i.e. 3.5, than a typical PWR $17 \times 17$ lattice with $\mathrm{H} / \mathrm{HM}$ of 3.9 due to the higher $\mathrm{HM}$ density of the $\mathrm{U}_{3} \mathrm{Si}_{2}$ fuel. Although under-moderated in terms of neutronic performances, both the $19 \times 19$ and $17 \times 17$ designs have similar moderator to fuel volumetric ratio of $\sim 2$, and therefore the $19 \times 19$ lattice design poses no issues in normal and accidental operations. The main geometric parameters and fuel design characteristics are shown in Table 1.

The 3-batch $\mathrm{I}^{2} \mathrm{~S}$-LWR core loading pattern as shown in Figure 1 is identical to the one adopted by reference [11]. There are 40 fresh assemblies per reload out of 121 assemblies. The twice-burnt assemblies are positioned at
Table 1. Main fuel assembly design parameters.

\begin{tabular}{ll}
\hline Parameter & Value \\
\hline Lattice type & $19 \times 19$, square \\
Cladding material & Advanced SS (FeCrAl) \\
Fuel rods per assembly & 336 \\
Fuel pellet material & $\mathrm{U}_{3} \mathrm{Si}_{2}$ \\
Fuel rod outer diameter (in) & 0.36 \\
Cladding thickness (in) & 0.016 \\
Pellet-clad gap width (in) & 0.006 \\
Pellet outer diameter (in) & 0.316 \\
Pellet inner void diameter (in) & 0.1 \\
Fuel pellet dishing (\%) & 0.3 \\
Fuel density (\% of theoretical) & 95.5 \\
Fuel rod pitch (in) & 0.477 \\
\hline
\end{tabular}

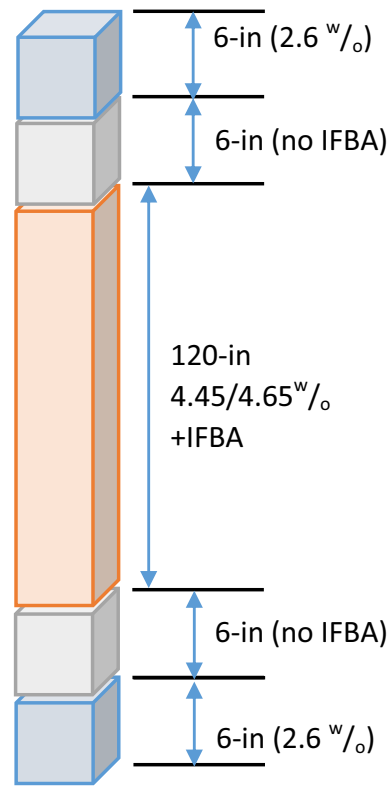

IFBA rods

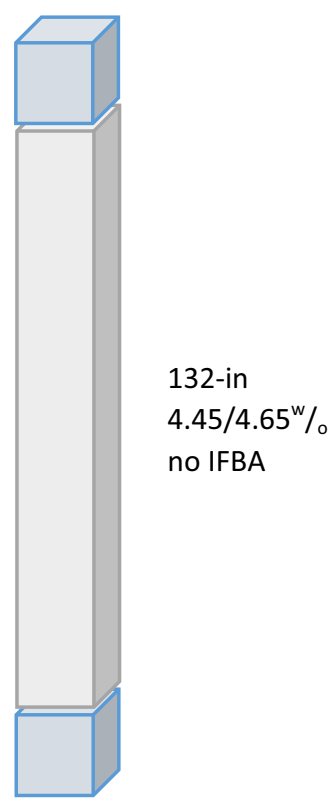

Non IFBA rods
Fig. 2. $I^{2} \mathrm{~S}-\mathrm{LWR}$ fuel axial stack.

the outermost peripheral locations to create a low leakage core. The $\mathrm{I}^{2} \mathrm{~S}-\mathrm{LWR}$ features 45 reactivity control clusters assemblies with 24 control rods (Ag-In-Cd) in the assembly.

The $\mathrm{U}_{3} \mathrm{Si}_{2}$ core design includes fresh and burned assemblies as shown in Figure 1. Fresh assemblies exploit different enrichments (i.e. $4.65,4.45$ and $2.6 \mathrm{w}$ o) . The active core height of the $\mathrm{I}^{2} \mathrm{~S}-\mathrm{LWR}$ fuel axial stack is presented in Figure 2. In fuel assemblies with integral fuel burnable absorber (IFBA) rods (Fig. 2), only the middle portion (120-in) contains $\mathrm{ZrB}_{2}$ burnable poison, which is surrounded by 6 -in non-IFBA top and bottom layers carrying the same fuel enrichment. Finally, 6-in top and bottom axial blankets are used to create the fuel stack. Lower enrichment $(2.6 \mathrm{w} / \mathrm{o})$ is used in the blankets in order to decrease the axial leakage of neutrons. 


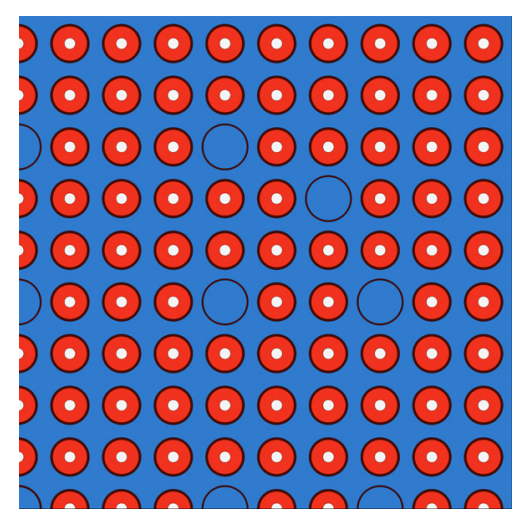

a. No IFBA rods

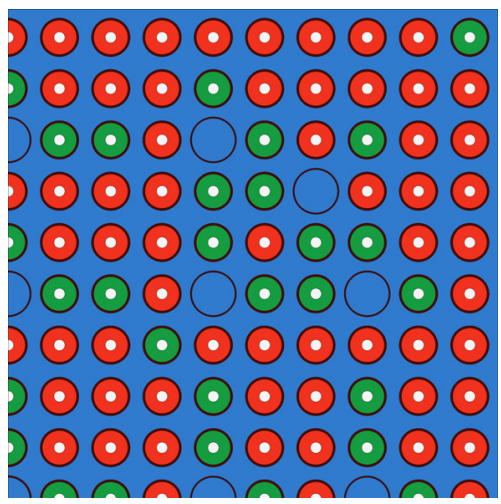

c. 100 IFBA rods

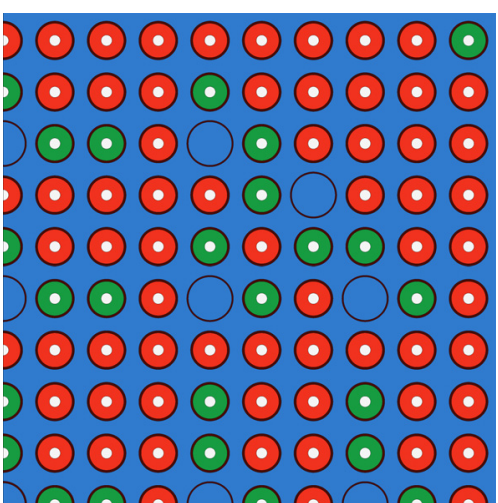

b. 84 IFBA rods

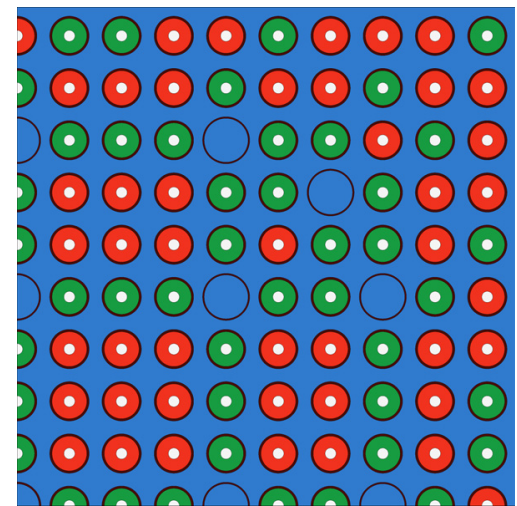

d. 156 IFBA rods

Fig. 3. $\mathrm{I}^{2} \mathrm{~S}-L W R$ IFBA loading patterns - the top right quadrant of the assembly is shown; IFBA rods are indicated in green.

The ${ }^{10} \mathrm{~B}$ concentration used in the IFBA rods for the $\mathrm{I}^{2} \mathrm{~S}$ LWR, with $\mathrm{U}_{3} \mathrm{Si}_{2}$, fuel design, is $2.5 \mathrm{mg} /$ in. Four assembly loading patterns are used to flatten the core power distribution and were investigated here, as depicted in Figure 3 .

\subsection{Methods}

The current work was divided into the following stages:

- verification of the 2D WIMS assembly models against the reference solutions obtained with the Monte Carlo (MC) code Serpent. Serpent is a continuous-energy MC reactor physics code recently developed for reactor physics applications at VTT Technical Research Centre of Finland. Serpent can be used for 2D fuel lattice calculations as well as for 3D full core simulations. JEFF-3.1 cross-section libraries were used for WIMS and Serpent to minimize discrepancies in neutronic parameters $\left(\mathrm{e} . \mathrm{g} . \mathrm{k}_{\mathrm{inf}}\right)$ that could arise from the use of different nuclear data evaluations;

- the core physics analysis of the $\mathrm{I}^{2} \mathrm{~S}-\mathrm{LWR}$ core design was performed with the core physics package PANTHER. WIMS10 was used for lattice data generation by employing a 172-group JEFF-3.1-based library. WIMS10 utilizes a multicell collision probability method to form 22-group cross-sections, followed by a method-of-characteristics solution to generate data for PANTHER. Results were compared to those reported in reference [11], which use deterministic lattice calculates to provide data for a 3D core analysis $[27,28]$. PANTHER used the same 3-batch self-generating reloading scheme that was iteratively applied to the $\mathrm{U}_{3} \mathrm{Si}_{2}$ core design until the main core parameters converged and a 12-month equilibrium cycle was reached.

\subsection{Results}

\subsubsection{WIMS vs. Serpent comparison}

This section presents the single-assembly comparison between WIMS and Serpent for different fuel assembly layouts (i.e. different numbers of IFBA rods). Figure 4 shows criticality curves for the different cases examined. Zero buckling hypothesis was adopted in the current comparison. The difference in reactivity, between Serpent and WIMS, for each of the cases is presented in Figure 5. In addition, Figure 6 shows the maximum difference in withinassembly power (pin-by-pin) between Serpent and WIMS. It must be pointed out that the average absolute difference in the assembly power between the codes is much lower $(<0.15 \%)$. Figure 7 presents the pin-by-pin power distribution for an assembly that carries 156 IFBA rods. 


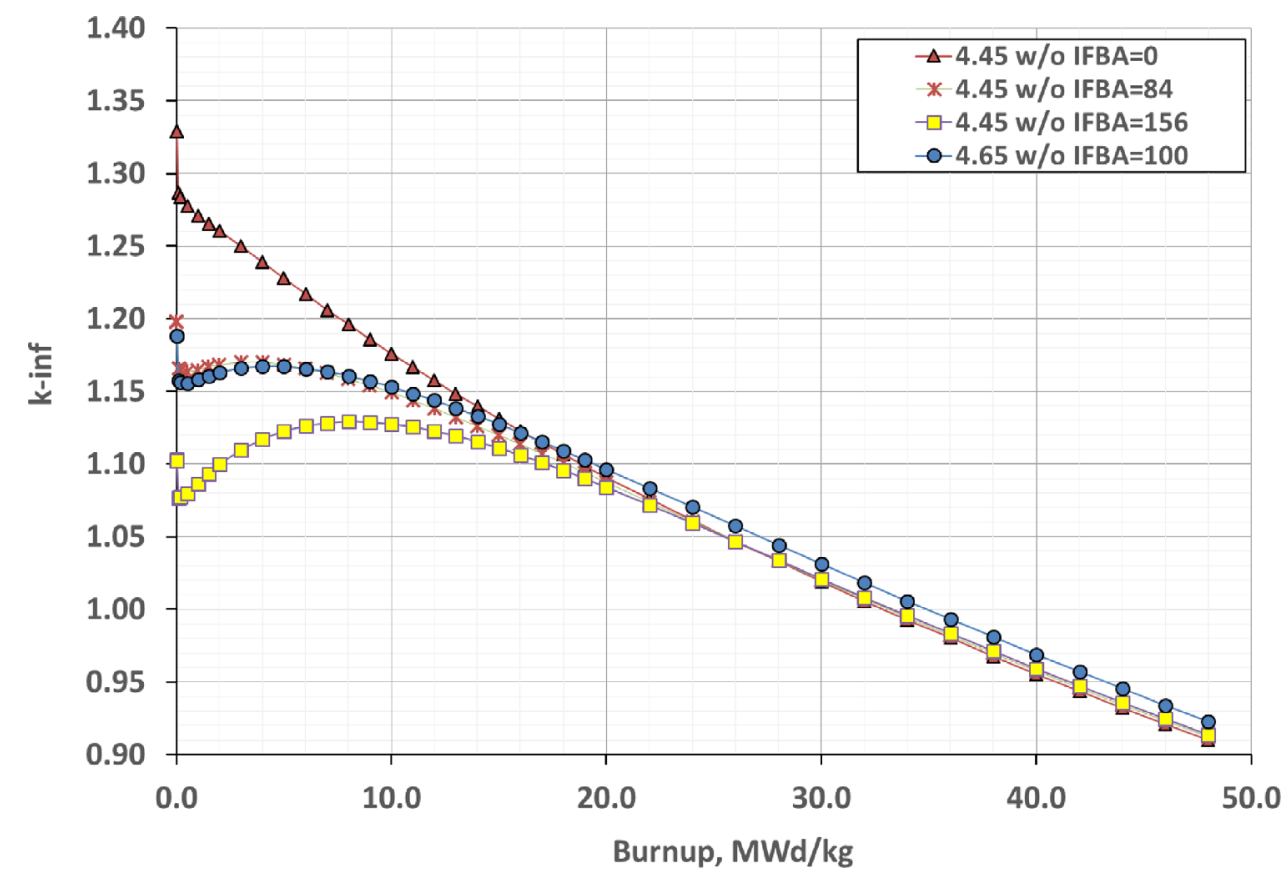

Fig. 4. Criticality curves for different IFBA loading patterns (note that k-inf initially increases with burnup as the burnable poison burns out).

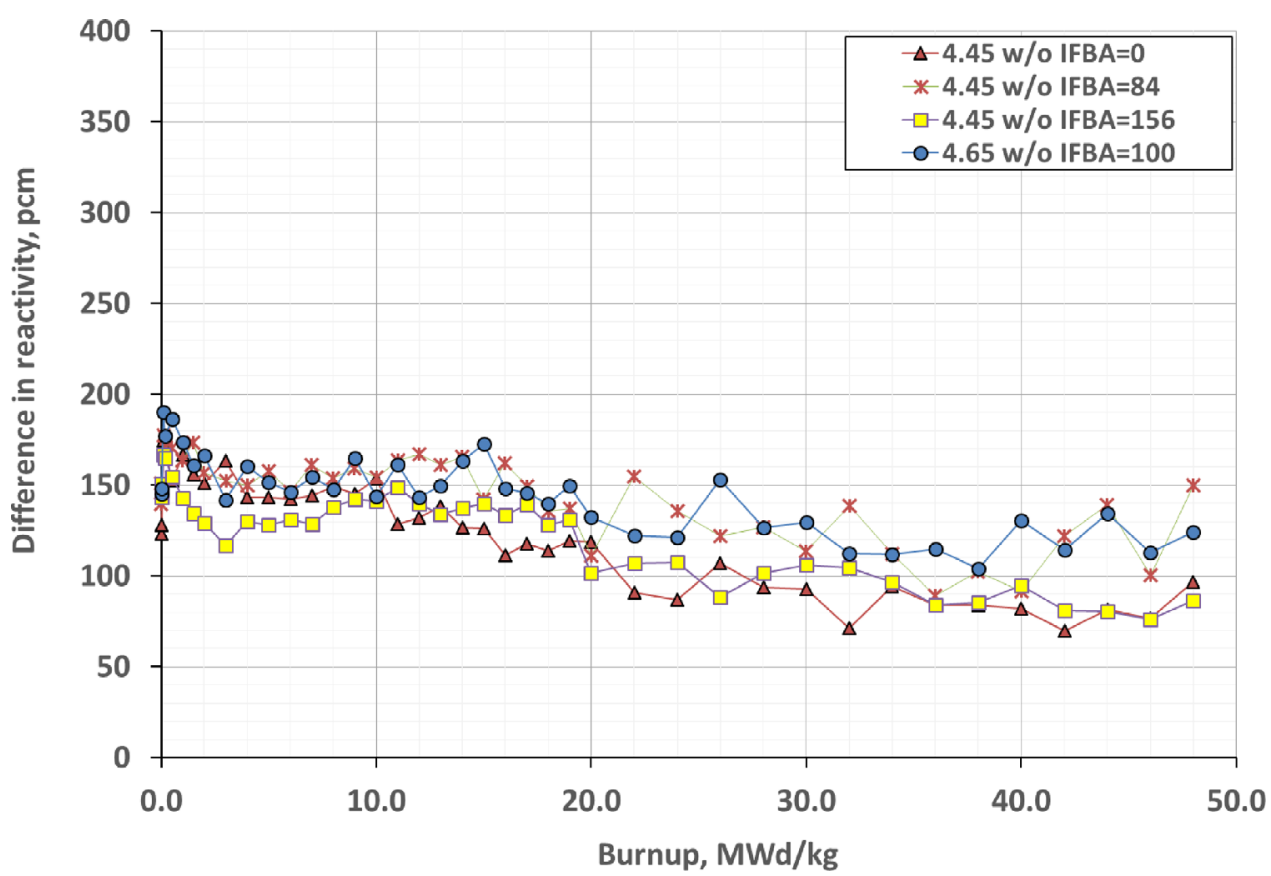

Fig. 5. Difference in reactivity (WIMS vs. Serpent) for different IFBA loading patterns.

\subsubsection{Equilibrium core analysis}

The representative burnup (MWD/t $\mathrm{t}_{\mathrm{HM}}$ ) distribution at the beginning of the equilibrium cycle is presented in the octant-core map in Figure 8. Figure 9 shows the required boron concentration to maintain criticality over the equilibrium cycle. The radial and total power peaking factors, which represent the quarter-wise assembly values, are depicted in Figure 10, which also presents the timedependent axial offset. Results are in good agreement with the values reported in reference [11] (e.g. assembly burnups within around 1\%). This cross-comparison of results provides enhanced confidence in the reliability and accuracy of the results. 


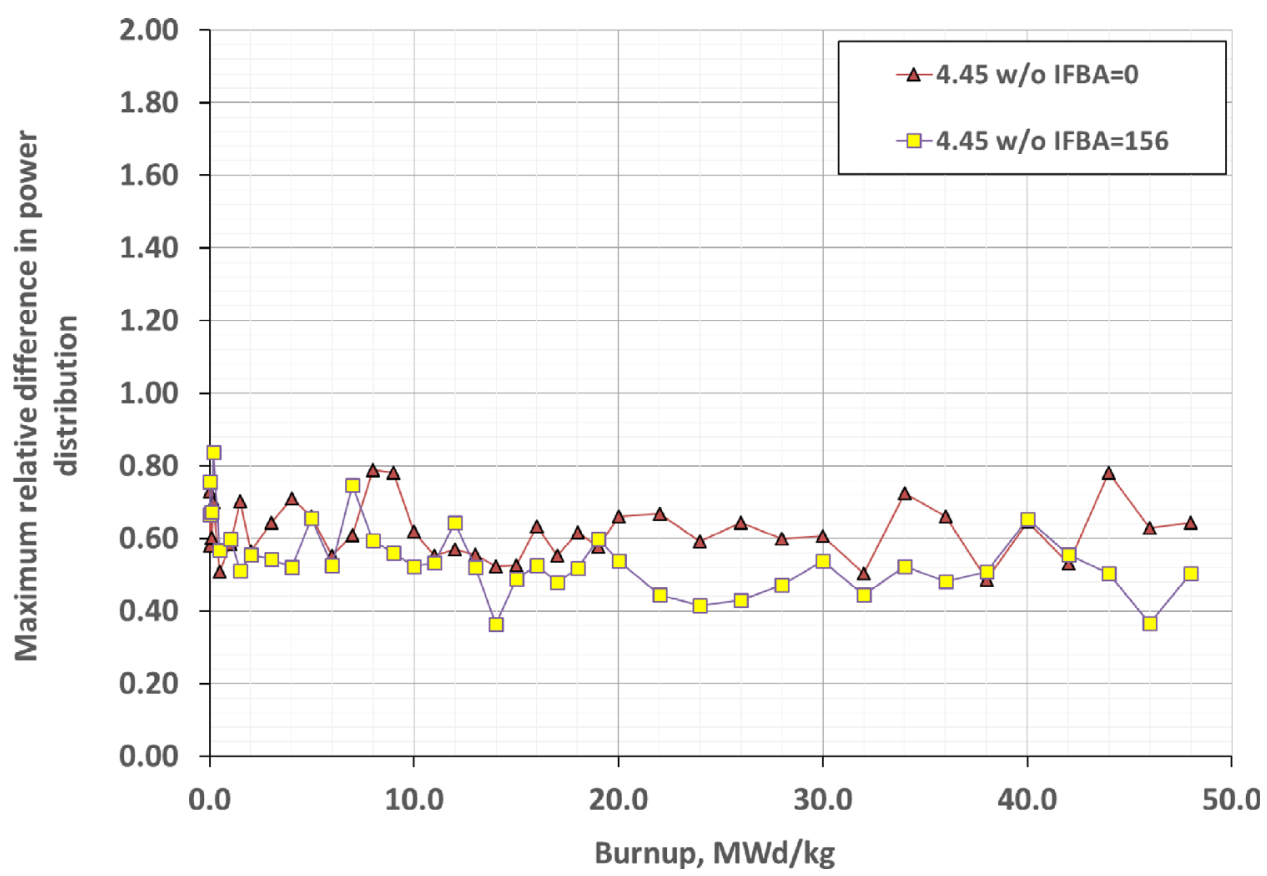

Fig. 6. Maximum relative difference (\%) in assembly radial power distribution (WIMS vs. Serpent).

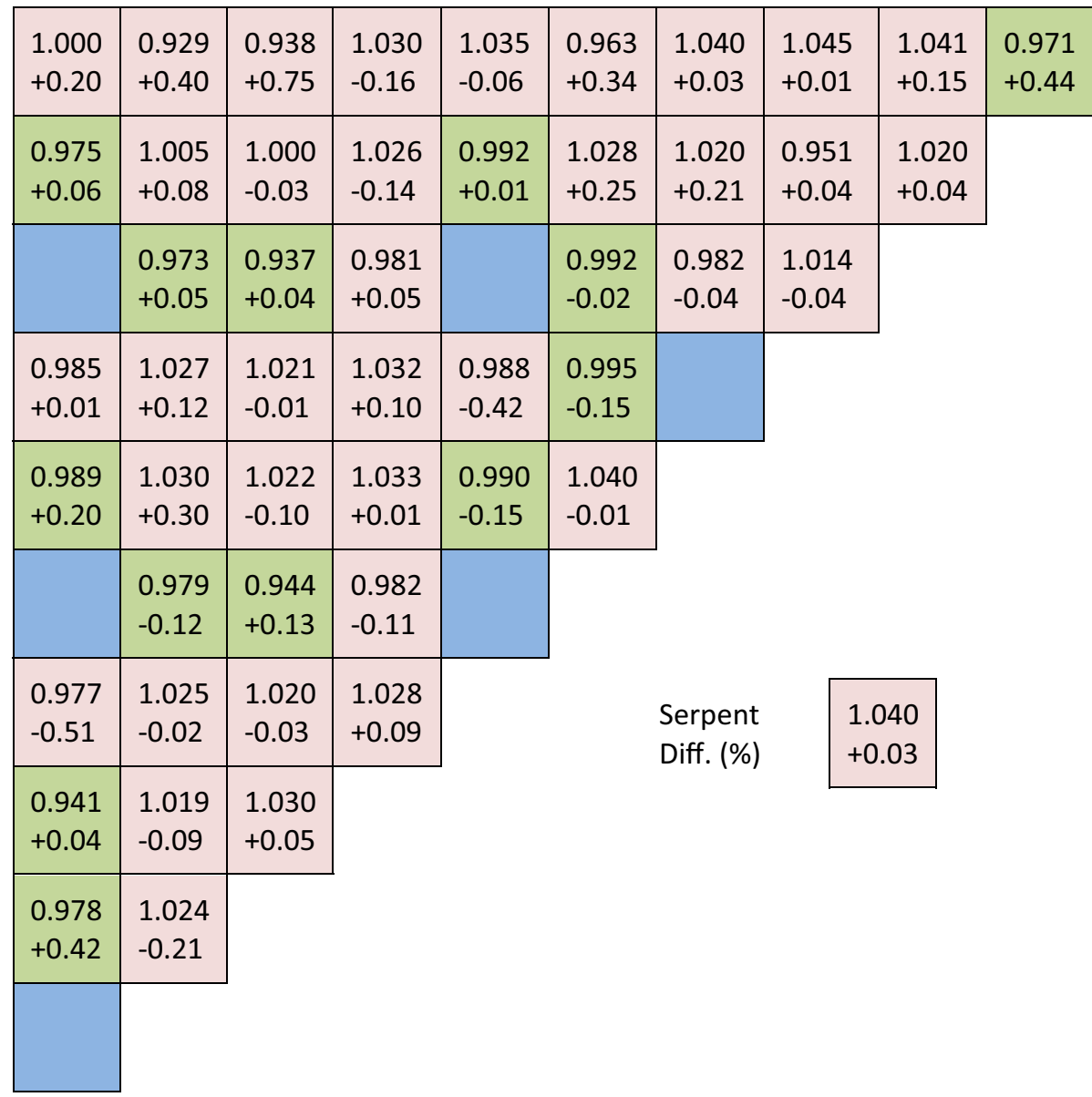

Fig. 7. Top right octant assembly $(4.45 \mathrm{w}$ o and 156 IFBA rods) radial power distribution (WIMS vs. Serpent) at zero burnup; IFBA rods are indicated in green. 


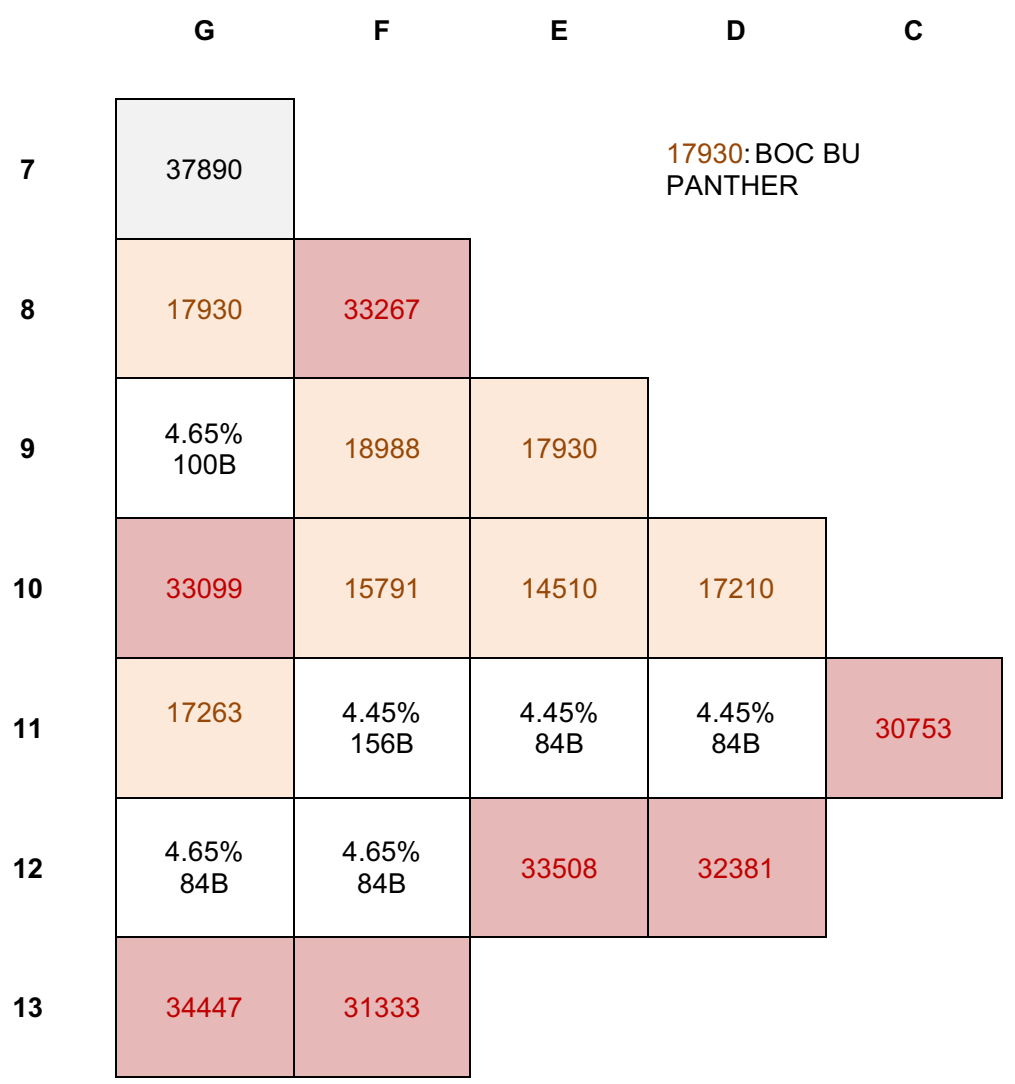

Fig. 8. $\mathrm{I}^{2} \mathrm{~S}-\mathrm{LWR}$ equilibrium burnup in PANTHER.

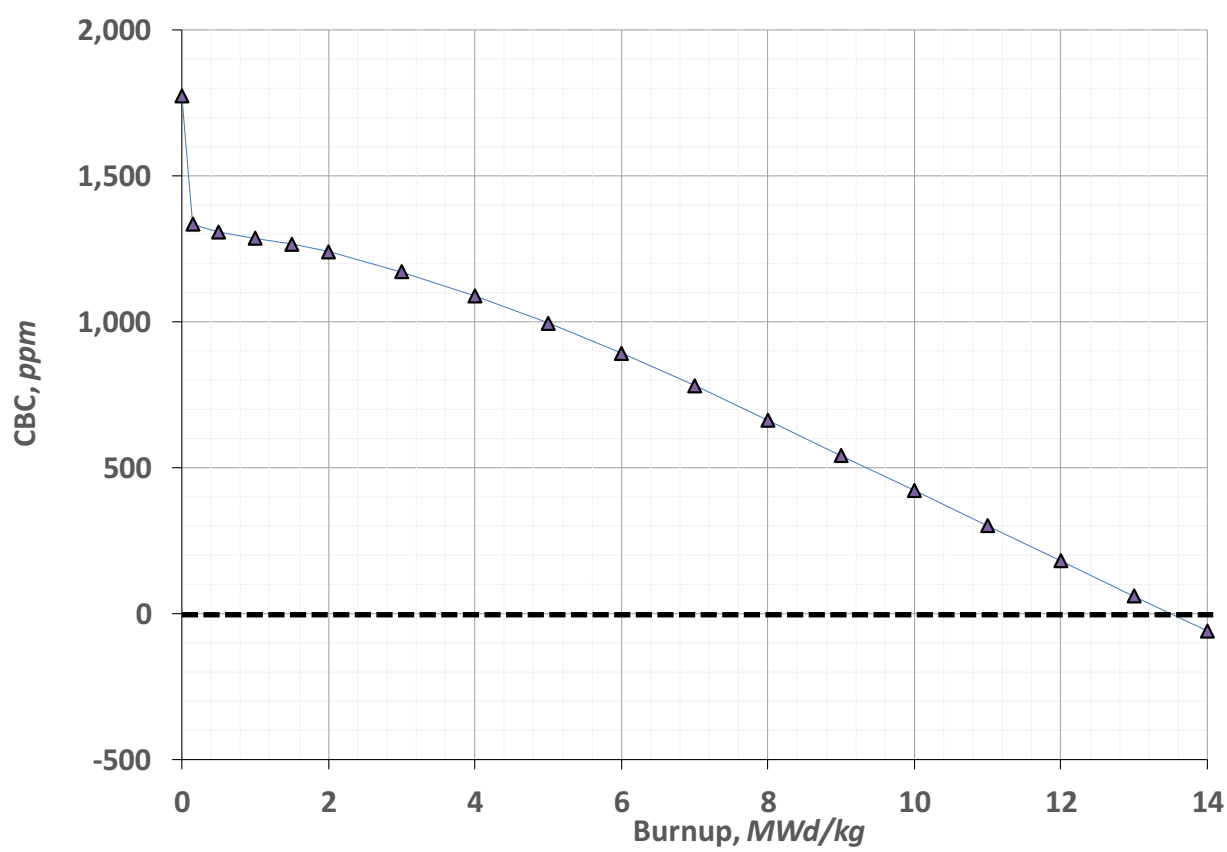

Fig. 9. Comparison of the critical boron concentration (ppm) as a function of burnup in PANTHER. 

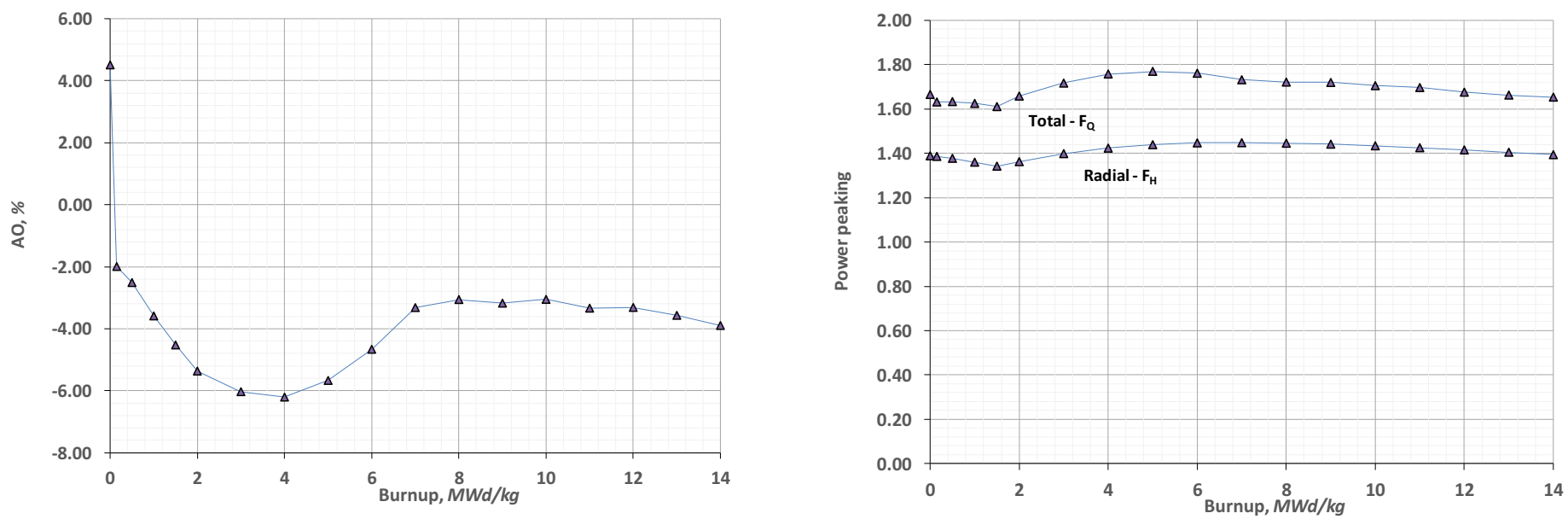

Fig. 10. Axial Offset (AO) (left) and radial and total power peaking factors (right) for $\mathrm{I}^{2} \mathrm{~S}-\mathrm{LWR}$ calculated using PANTHER.

\section{Conclusions}

The UK has a long history in industrial fuel manufacture and fabrication for a wide range of reactor systems including LWRs. This is supported by a national infrastructure to perform experimental and theoretical R\&D in fuel performance, fuel transient behaviour and reactor physics. The ANSWERS lattice code WIMS and core simulator PANTHER are used to support the operation of existing PWRs, including in the UK and Belgium. Modelling of ATFs is a natural extension of these capabilities and can largely be performed using existing calculation routes. Reactor physics modelling of the $\mathrm{I}^{2} \mathrm{~S}-$ LWR equilibrium cycle core was performed with the WIMS-PANTHER codes. The results were compared to reported results for the equilibrium cycle of the $\mathrm{I}^{2} \mathrm{~S}-\mathrm{LWR}$ and indicate that there is a reasonable agreement between the codes. One possible source for the observed deviations between the codes is the different cross-section library employed in WIMS to generate lattice parameters. For this study, the JEFF-3.1 libraries were used in WIMS, whereas ENDF BVII.0 was used in reference [11]. Future work could consider using the ENDF BVII.0 library in WIMS to allow for a more consistent comparison. It may also ultimately be necessary to validate the reactor physics codes against experimental data.

We are grateful to our colleagues in the ANSWERS team for providing advice and guidance during the preparation of this paper.

This research was in part funded by the UK Engineering and Physical Sciences Research Council (EPSRC) under grant EP/ K033611/1 and by the US Depart of Energy (DOE) Office of Nuclear Energy's Nuclear Energy University Programs (NEUP).

\section{References}

1. F. Franceschini, E.J. Lahoda, Advanced fuel developments to improve fuel cycle cost in PWR, in GLOBAL 2011, Makuhari, Japan, Dec. 11-16, 2011 (2011)
2. K.E. Metzger, T.W. Knight, R.L. Williamson, Model of $\mathrm{U}_{3} \mathrm{Si}_{2}$ fuel system using bison fuel code, in ICAPP 2014, Charlotte, NC, USA, Apr. 6-9, 2014 (2014)

3. H. Kim, I. Kim, Y. Koo, J. Park, Application of coating technology on zirconium-based alloy to decrease hightemperature oxidation, in 17th International Symposium on Zirconium in the Nuclear Industry, Andhra Pradesh, India (2013)

4. A. Abe, C. Giovedi, D.S. Gomes, A.T. Silva, Revisiting stainless steel as PWR fuel rod cladding after Fukushima Daiichi accident, J. Energy Power Eng. 8, 973 (2014)

5. E.J. Lahoda, F. Franceschini, Advanced fuel concepts RTTR-11-12 (Westinghouse Electric Company LLC, 2011)

6. Westinghouse Electric Company LLC, Enhancing safety: the pursuit of accident tolerant fuel, [Online], Available: http:// www.westinghousenuclear.com/About/News/Features/ View/ArticleId/481/Enhancing-Safety-The-Pursuit-of-Acci dent-tolerant-Fuel [Accessed 20.2.2015]

7. T. Newton et al., Developments within WIMS10, in PHYSOR 2008, Interlaken, Switzerland, Sept. 14-19, 2008 (2008)

8. E.A. Morrison, PANTHER User Guide E/REP/BBDB/0015/ GEN/03 ED/PANTHER/UG/5.5, British Energy, 2003

9. B. Petrovic, Integral inherently safe light water reactors $\left(\mathrm{I}^{2} \mathrm{~S}-\right.$ LWR) concept: extending SMR safety features to large power output, in ICAPP 2014, Charlotte, NC, USA, Apr. 6-9, 2014 (2014)

10. J. Leppänen, M. Pusa, T. Viitanen, V. Valtavirta, T. Kaltiaisenaho, The Serpent Monte Carlo code: status, development and applications in 2013, Ann. Nucl. Energy 82, 142 (2015)

11. D. Salazar, F. Franceschini, $\mathrm{I}^{2} \mathrm{~S}-\mathrm{LWR}$ equilibrium cycle core analysis, in PHYSOR 2014, Kyoto, Japan, Sept. 29-Oct. 3, 2014 (2014)

12. Department of Energy \& Climate Change, Nuclear R\&D Accident Tolerant Fuel: Grant Notification, 2014

13. Department of Energy and Climate Change, DECC Science Advisory Group: Horizon Scanning, October 2013

14. Nuclear Innovation and Research Advisory Board, NIRAB Annual Report, 2014

15. J.L. Hutton et al., Comparison of WIMS/PANTHER calculations with measurement on a range of operating PWR, in PHYSOR 2000, Pittsburgh, USA, May 2000 (2000) 
16. S. Alam, B.A. Lindley, G.T. Parks, Feasibility study of the design of homogeneously mixed thorium-uranium, in ICAPP 2015, Nice, France, May 3-6, 2015 (2015)

17. C. Harrington, Reactor Physics Modelling of the Shippingport Light Water Reactor, MPhil Dissertation, University of Cambridge, 2012

18. S.F. Ashley et al., Fuel cycle modelling of open cycle thoriumfuelled nuclear energy systems, Ann. Nucl. Energy 69, 314 (2014)

19. T. Fei, E.A. Hoffman, T.K. Kim, T.A. Taiwo, Performance evaluation of two-stage fuel cycle from SFR to PWR, in GLOBAL 2013, Salt Lake City, UT, USA (2013)

20. F. Heidet, T.K. Kim, T.A. Taiwo, Two-stage fuel cycles with accelerator-driven systems, in PHYSOR 2014, Kyoto, Japan, Sept. 28-Oct. 3, 2014 (2014)

21. L.L. Snead et al., Handbook of $\mathrm{SiC}$ properties for fuel performance modelling, J. Nucl. Mater. 371, 329 (2007)

22. Z. Xu, Design strategies for optimizing high burnup fuel in pressurized water reactors, $\mathrm{PhD}$ Thesis, Massachusetts Institute of Technology, 2003
23. G.T. Parks, Pressurised water reactor fuel management using PANTHER, Nucl. Sci. Eng. 124, 178 (1996)

24. G.T. Parks, M.P. Knight, Loading pattern optimization in hexagonal geometry using PANTHER, in PHYSOR 96, Mito, Japan, Sept. 16-20, 1996 (1996)

25. N.Z. Zainuddin, B.A. Lindley, G.T. Parks, Towards optimal in-core fuel management of thorium-plutonium-fuelled PWR cores, in ICONE 21, 21st International Conference on Nuclear Energy, Chengdu, China, July 29-Aug. 3, 2013 (2013)

26. B.A. Lindley, A. Ahmad, N.Z. Zainuddin, F. Franceschini, G. T. Parks, Steady-state and transient core feasibility analysis for a thorium-fuelled reduced-moderation PWR performing full transuranic recycle, Ann. Nucl. Energy 72, 320 (2014)

27. M. Ouisloumen et al., PARAGON: The New Westinghouse Assembly Lattice Code, in ANS Int. Mtg. on Mathematical Methods for Nuclear Applications, Salt Lake City, UT, USA (2001)

28. L. Mayhue et al., Qualification of NEXUS/ANC nuclear design system for PWR analyses, in PHYSOR 2008, Interlaken, Switzerland, Sept. 16-19, 2008 (2008)

Cite this article as: Benjamin A. Lindley, Dan Kotlyar, Geoffrey T. Parks, John N. Lillington, Bojan Petrovic, Reactor physics modelling of accident tolerant fuel for LWRs using answers codes, EPJ Nuclear Sci. Technol. 2, 14. (2016) 